\title{
Commentary: Is donor acceptance a bigger problem than donor availability? Time for a realistic look
}

\author{
James D. St Louis, MD, ${ }^{\mathrm{a}}$ and Mark Plunkett, $\mathrm{MD}^{\mathrm{b}}$
}

\footnotetext{
From the ${ }^{a}$ Department of Surgery, University of Missouri-Kansas City School of Medicine, Kansas City, Mo; and ${ }^{\mathrm{b}}$ Congenital Heart Center at Children's Hospital of Illinois, Peoria, Ill. Disclosures: Authors have nothing to disclose with regard to commercial support.

Received for publication July 6, 2019; revisions received July 6, 2019; accepted for publication July 8, 2019; available ahead of print Oct 22, 2019.

Address for reprints: James D. St Louis, MD, Children's Mercy Kansas City, 2401 Gillham Rd, Kansas City, MO 64108 (E-mail: jdstlouis@cmh.edu).

J Thorac Cardiovasc Surg 2019;158:1663-4

$0022-5223 / \$ 36.00$

Copyright (C) 2019 by The American Association for Thoracic Surgery

https://doi.org/10.1016/j.jtcvs.2019.07.151
}

Overall morbidities and mortalities associated with pediatric heart transplantation have significantly improved during the last several decades, particularly with improvements in the management of transplant-related rejection. ${ }^{1}$ The International Society of Heart and Lung Transplantation currently reports an overall improvement in posttransplant survival to $75 \%$ at 10 years. $^{2}$ Factors critical to this improvement include more aggressive listing of recipient candidates, improved surgical techniques, and better understanding of posttransplant management, particularly evolving antirejection protocols. It is therefore quite understandable that, with these improvements, the number and complexity of patients being referred for cardiac transplantation have increased dramatically during the last decade. Certain subgroups of patients who were never previously considered for listing, particularly those with failing single-ventricle palliations, now routinely make up a substantial portion of pediatric heart transplant wait lists.

An Achilles' heel of pediatric transplantation is the persistent stagnation of donor availability, predominately with the younger donor pool failing to match the increase in numbers of patients being placed on a wait list. Current data from the United Network for Organ Sharing suggest that there has been no significant increase in donor availability for most age groups. With an average wait list time for 4 to 6 months and the lack of donors to match need, it is thus no wonder that wait list mortality has remained constant at $25 \%$ during the last decade. ${ }^{3}$ This mortality continues to be the highest among all other solid organ transplant populations. Of course, this reflects a multitude of issues; however, the ongoing shortage of organs, including the possible underutilization of available donors, remains a glaring challenge.

In this edition of The Journal of Thoracic and Cardiovascular Surgery, Riggs and associates ${ }^{4}$ present a retrospective, population-based analysis of the effect on results of body weight mismatch between donors and recipient candidates for pediatric heart transplantation. The data source for this analysis was the United Network for Organ Sharing, with

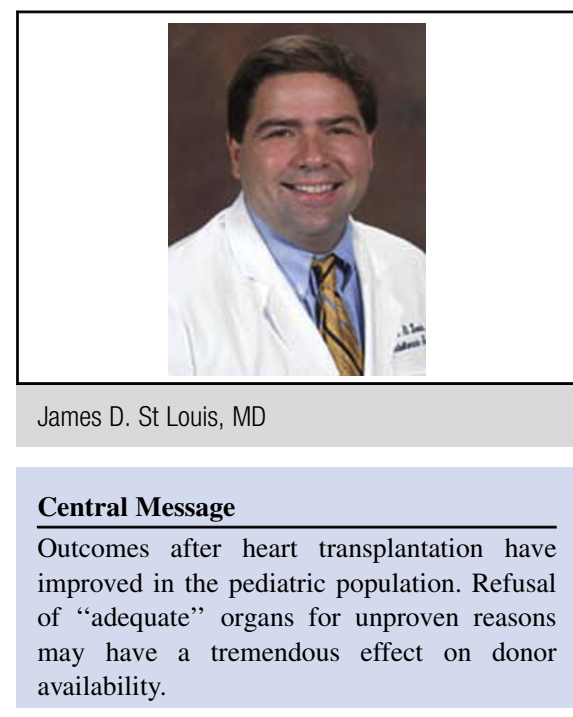

See Article page 1652 .

more than 7000 patients included. The study revealed significant variation in the use of weight mismatching as a criterion for refusal of a donor organ. Of greater importance, the study concluded that when a donor was accepted and there existed a significant weight mismatch between donor and recipient, survival was not affected relative to transplantation with a matched donor and recipient.

In 2015 , nearly $55 \%$ of possible pediatric donor hearts were not used for transplantation. Although this may partially reflect the aggressive mission of our organ procurement establishments to list and place all potential donors regardless of clinical status, equally important are the perceptions and biases of the institutional protocols concerning the criteria for acceptance or refusal of organs. The authors of the current article published a previous analysis ${ }^{5}$ in which they assessed the effect on responses to organ offers that had been turned down for "quality" by other institutions. Amazingly, they concluded that transplant outcomes were not affected by the number of refusals any one donor experienced. Numerous studies have revealed that a significant amount of decision-making abilities and judgments are formed by factors unrelated to objective clinical outcomes analysis. Much of what forms decision-making skills is based on one's educational experience, anecdotal clinical experiences, perceptions acquired from senior colleagues, and, at times, unsubstantiated fear of poor outcomes related to a particular decision. The age-old adage that medicine is 
more an art form than an actual science has long influenced the clinical management of many disease processes. Although we can say with certainty that this modus operandi has been successful in treating several millennia of ill patients, we have a duty to our profession and patients to apply the most stringent scientific processes to support the potential life and death decisions applied to a population of patients who have never been considered for such treatment. The field of organ replacement therapy has advanced to a place that was previously inconceivable. Prolonged periods waiting for the "perfect" organ, while allowing significant numbers of "adequate" organs to go to waste, can no longer be accepted as standard practice.

\section{References}

1. Dipchand A. Current state of pediatric cardiac transplantation. Ann Cardiothorac Surg. 2017;7:31-55.

2. The registry of the International Society of Heart and Lung Transplantation: nineteenth pediatric heart transplantation report-2016; focus theme: primary diagnostic indications for transplant. J Heart Lung Transplant. 2016;35: $1185-95$.

3. Almond CSD, Thiagarajan RR, Piercey GE, Gauvreau K, Blume ED, Bastardi HJ, et al. Waiting list mortality among children listed for heart transplantation in the United States. Circulation. 2009;119:717-27.

4. Riggs KW, Giannini CM, Szugye N, Woods J, Chin C, Moore RA, et al. Time for evidence-based, standardized donor size matching for pediatric heart transplantation. J Thorac Cardiovasc Surg. 2019;158:1652-60.

5. Rizwan R, Zafer F, Bryant R III, Tweddell JS, Lorts A, Chin C, et al. The number of refusals for donor organ quality does not impact heart transplant outcomes in children. Ann Thorac Surg. 2018;105:1223-31. 